\title{
The role of echocardiography in differential diagnosis of cardiac sources of embolism
}

\section{Kristina Selthofer- Relatić ${ }^{*}$}

University of Osijek School of Medicine, University Hospital Centre Osijek, Osijek, Croatia
KEYWORDS: cardiac embolism, echocardiography, stroke.

CITATION: Cardiol Croat. 2017;12(4):145. I https://doi.org/10.15836/ccar2017.145

*ADDRESS FOR CORRESPONDENCE: Kristina Selthofer-Relatić, Klinički bolnički centar Osijek, J. Huttlera 4, HR-31000 Osijek, Croatia. / Phone: +385-31-511-717 / E-mail: selthofer.relatic@gmail.com

ORCID: Kristina Selthofer-Relatić, http://orcid.org/0000-0002-9890-6489

IIIIIIIIIIIIIIIIIIIIIIIIIIIIIIIIIIIIIIIIIIIIIIIIIIIIIIIIIIIIIIIIIIIIIIIIIIIIIIIIIIIIIIIIIIIIIIIIIIIIIIIIIIIIIIIIIIIIII

Stroke is a leading cause of morbidity and mortality. According to pathophysiologic mechanisms, stroke can be divided into two major groups: ischemic (85\%) and hemorrhagic (15\%). $20 \%$ of ischemic strokes are caused by cardiac embolisms. Brain imaging (CT, MR) confirms the diagnosis, some specific characteristics seen during the imaging of the brain may suggest a distinct cardiac cause. Clinical history, examination and electrocardiogram can shed light on a potential cardiac cause of ischemic stroke. Echocardiography is a key method in the evaluation, diagnosis and management of embolic stroke (transthoracic echo, transesophageal echo, 3D echo, contrast echo)

The etiological factors of cerebrovascular events are: blood clots, tumor fragments, infected and noninfected vegetations, calcified particles, atherosclerotic debris. Cardiac sources of emboli can be divided according to embolic potential into a) high risk: intracardiac thrombi (atrial arrhythmias - atrial fibrillation, ischemic heart disease, non-ischemic cardiomyopathies, prosthetic valves and devices), intracardiac vegetations, intracardiac tumors, aortic atheroma; b) low risk: spontaneous echo contrast without clot, left ventricle aneurysm without clot, intracardiac calcifications, valvular anomalies, septal defects and anomalies.

The indications for echocardiography in patients with neurological events are: abrupt occlusion of a major peripheral or visceral artery in patients of any age; younger patients with CV events ( $<45$ years), older patients (>45 years) with neurologic events without evidence of cerebrovascular disease or other obvious cause, patients for whom a clinical therapeutic decision will depend on the results of echocardiography. 